\title{
Discontinuation of prolonged dual antiplatelet therapy for a dental extraction: A nearly-fatal decision
}

Stent thrombosis remains one of the most feared complications in patients with multiple stents implanted. In this clinical setting, optimal upfront dual antiplatelet therapy (DAPT) duration still remains controversial. Herein, we wish to report a case of life-threatening very-late stent thrombosis after DAPT temporary discontinuation, highlighting the prolonged increased thrombotic risk of complex angioplasty, even in otherwise low-ischemic risk patients according to recent recommendations. Our data are in line with novel information and might be useful in the pre-operative management of patients with complex percutaneous coronary intervention ( $\mathrm{PCI}$ ).

Keywords: Dual antiplatelet therapy - Percutaneous coronary intervention - Stable ischemic heart disease - Stent thrombosis - Cardiogenic shock - Glycoprotein Ilb/Illa inhibitors

\section{Case Report}

A 66-year-old hypertensive man presented with an acute anterior ST-elevation myocardial infarction (STEMI) and ensuing cardiogenic shock, five years after implantation of three drug-eluting (DES) stents in proximal left anterior descending (LAD), proximal first diagonal branch and circumflex artery (LCX) (Figures 1A-1D) for stable ischemic heart disease. The patient was asymptomatic since then; however, three days before his admission, he was advised to temporarily discontinue aspirin-clopidogrel DAPT for a minimal dental procedure (tooth extraction), and receive low molecular weight heparin (LMWH) as a five-day bridging therapy regimen.

The patient was transferred immediately to the catheterization laboratory; while coronary artery angiography (CAA) was in progress, however, he demonstrated devastating cardiogenic shock (noradrenaline dose $\leq 42$ $\mathrm{mcg} / \mathrm{min}$ ) and electrical storm (numerous episodes of ventricular tachycardia/ fibrillation), thus he was intubated and received several cycles of cardiopulmonary resuscitation and electrical cardioversion/ defibrillation. CAA disclosed total left main artery (LMA) occlusion with a Thrombolysis in Myocardial Infarction (TIMI) flow grade of 0 , implying generalized acute stent thrombosis (Figure 1A, Online Video 1). While the patient was in premortal shock-

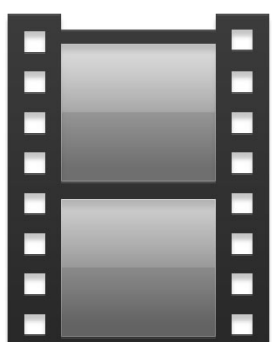

Online Video 1: Pre-interventional LAO coronary angiographic view with $30^{\circ}$ cranial angulation, revealing a total LMA occlusion with TIMI flow grade of 0 , as well as the three previously implanted drug-eluting stents in proximity to the lesion. $\mathrm{LAO}=$ left anterior oblique; $\mathrm{LMA}=$ left main artery; TIMI=Thrombolysis in Myocardial Infarction.
John Papanikolaou ${ }^{1,2 *}$, Nikolaos Platogiannis', Dionysios Gkekas', Nikolaos Barmpatzas', Konstantinos Spathoulas', Dimitrios Platogiannis ${ }^{1}$ 'Department of Cardiology, General Hospital of Trikala, Thessaly, Greece 2Department of Critical Care, University Hospital of Larissa, Thessaly, Greece

*Author for correspondence:

Tel: +302410682960

Fax: +302410670838

y_papanikolaou@hotmail.com Submitted: June 08, 2017 Accepted: July 05, 2017 Published online: July 10, 2017 

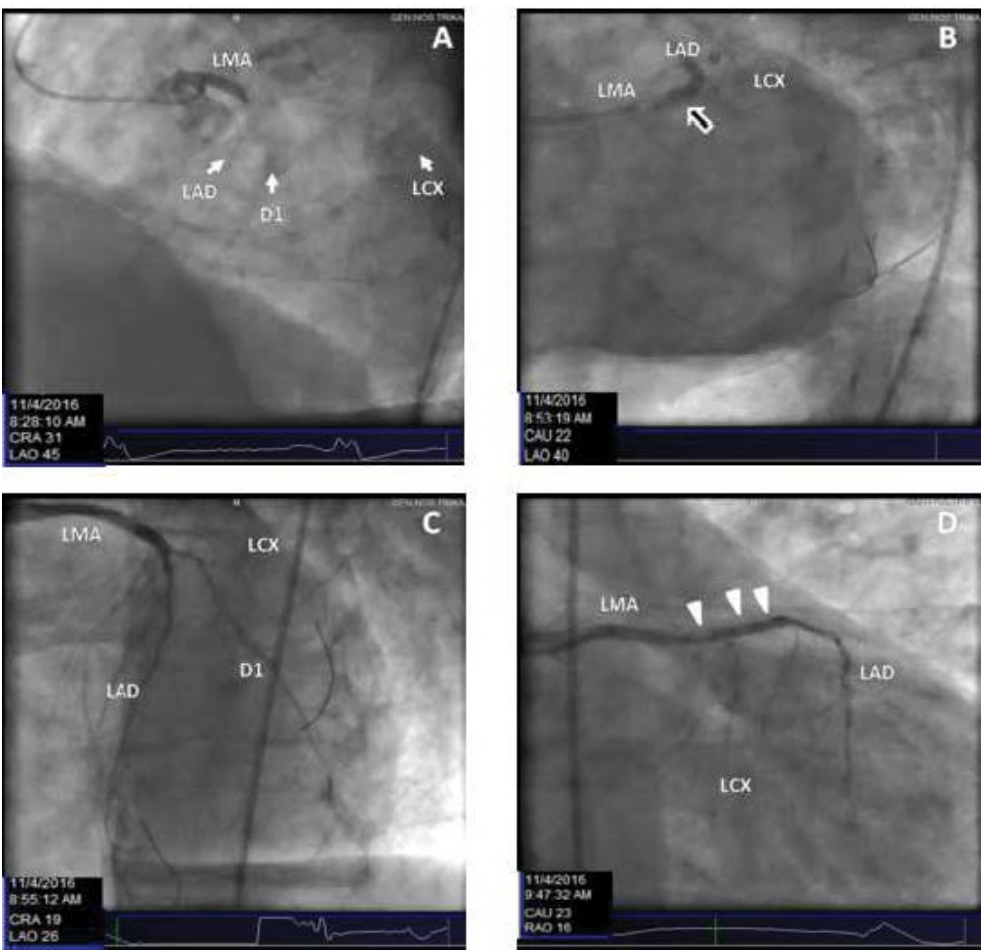

Figure 1: A. Pre-interventional coronary angiography illustrating a total LMA occlusion (TIMI flow grade of 0 ), as well as the three previously implanted DES in proximal LAD, proximal D1 and LCX (white arrows). B. DES placement within distal LMA/proximal LAD (white/black arrow). C,D. Post-interventional coronary angiographic views revealing LAD recanalization with extensive filling defects in its periphery (white arrowheads), yet still an obstructed LCX. LMA: Left Main Artery; TIMI: Thrombolysis in Myocardial Infarction; DES: Drug-eluting Stent; LAD: Left Anterior Descending artery; D1: first diagonal branch; LCX: Circumflex Artery; LAO: Left Anterior Oblique View; RAO: Right Anterior Oblique view; CRA: Cranial Angulation; CAU: Caudal Angulation.

condition, two overlapping $3.5 \times 18 \mathrm{~mm}$ Resolute $^{\mathrm{TM}}$ zotarolimus-eluting stents (Medtronic Inc., Santa Rosa, CA, USA) were successfully placed at distal LMA/ proximal LAD (Figure $1 \mathrm{~B}$ ), resulting in a TIMI flow grade of 3 in LAD, yet extensive LAD thrombotic remnants as well as a TIMI flow grade of 0 in LCX (Figures 1C and 1D).

The patient was given $600 \mathrm{mg}$ clopidogrel and 500 $\mathrm{mg}$ aspirin, tirofiban as bail-out therapy $(25 \mathrm{mcg} /$ $\mathrm{kg}$ five-minute bolus, followed by $0.15 \mathrm{mcg} / \mathrm{kg} / \mathrm{min}$ $18 \mathrm{~h}$ maintenance dose) and inotropic support with levosimendan $(0.05 \mu \mathrm{g} / \mathrm{kg} / \mathrm{min} 3$-day infusion $)$, and was transferred to coronary ICU. Remarkably, cardiac function was promptly restored (left ventricular ejection fraction was approximately $20 \%$ on ICU-admission, $>50 \%$ on ICU day-3), while central hemodynamics, renal function and lacticemia were also gradually improved; he was successfully extubated five days later, and discharged home in an excellent clinical condition, on medical therapy and aspirin-clopidogrel DAPT.

Our unique patient had previously received multiple stents for stable ischemic heart disease [1]. Apart from extensive coronary artery disease and greater stent length, no additional factors associated with increased ischemic/thrombotic risk were present [1]. According to recent recommendations [1], our patient summarized a DAPT score of 1 , which constitutes an unfavorable benefit/risk ratio for prolonged DAPT. In such circumstances, current evidence [1,2] questions the role of prolonged (beyond 6-12 months) DAPT, as protection against thrombosis may be outweighed by increased hemorrhagic risk. However, our rare patient was in low-bleeding risk [1]. In addition, he had a history remarkable for increased procedural PCI complexity (three stents implanted, three lesions treated, total stent length $>60 \mathrm{~mm}$ ); in this subset of patients, novel data provides evidence that longitudinal DAPT may bear a significant clinical benefit [3]. In line with the latter, our case highlights the critical role of complex PCI for very late stent thrombosis and stresses the importance of long-term DAPT in low-bleeding risk patients even in the absence of other predisposing ischemic risk factors.

Dental extractions are considered as low-hemorrhagic risk interventions that can safely be performed under dual or even single antiplatelet therapy [4,5]. Additionally, although recommended by several societies [6], heparin bridging therapy is probably ineffective due to its minor antiplatelet effects [7]. Bridging tTherapy wfit Th LMWH, 
similar to what is recommended for patients on warfarin, has also been proposed by several societies during temporary interruptions in antiplatelet treatment in patients with coronary stents who need surgery [6,7]. Despite its safe profile, however, heparin is probably ineffective in preventing acute stent thrombosis, due to its minor antiplatelet effect [6,7]. In this respect, our case points out that effort should be made minor surgical interventions to be performed under ongoing antiplatelet treatment, while LMWH as an alternative antithrombotic therapy may be ineffective and critically increase peri-procedural thrombotic danger.

Medical literature has recently focused on very late stent thrombosis after DES implantation; yet, there is only sparse evidence-based therapeutic data and even far less knowledge in the outcomes of patients with stent thrombosis-induced STEMI and shock. Certainly, case reports may be of less clinical value in advancing scientific knowledge and reviewing current therapeutic algorithms in rare diseases. Although thrombolysis has been successfully utilized for stent thrombosis-induced STEMI [8], our rare case ex juvantibus emphasize the vital importance of the availability of catheterization laboratory and primary PCI in the clinical setting of devastating cardiogenic shock. In addition, in the event of problematic primary PCI and angiographic evidence of intracoronary thrombotic remnants, our report highlights the pivotal role of glycoprotein IIb/ IIIa inhibitors as bailout therapy [9]. Finally, the non-catecholamine inotrope levosimendan may also play a crucial role in myocardial "salvage" in the extremely stressful condition of stent thrombosisinduced hemodynamic collapse; instead, exogenous catecholamines (e.g. dobutamine) may further precipitate the vicious-circle of catecholamine-mediated myocardial damage under such circumstances [10].

\section{Executive summary}

Stent thrombosis remains one of the most feared complications in patients with multiple stents implanted. In this clinical setting, optimal upfront dual antiplatelet therapy (DAPT) duration still remains controversial.

Herein, we wish to report a case of life-threatening very-late stent thrombosis after DAPT temporary discontinuation, highlighting the prolonged increased thrombotic risk of complex angioplasty, even in otherwise low-ischemic risk patients according to recent recommendations.

Our data are in line with novel information and might be useful in the pre-operative management of patients with complex percutaneous coronary intervention (PCI).

\section{References}

1. Levine GN, Bates ER, Bittl JA, et al. ACC/AHA Guideline Focused Update on Duration of Dual Antiplatelet Therapy in Patients With Coronary Artery Disease: A Report of the American College of Cardiology/American Heart Association Task Force on Clinical Practice Guidelines. J. Am. Coll. Cardiol. 68(10): 1082-1115 (2016).

2. Palmerini T, Della Riva D, Benedetto U, et al. Three, six, or twelve months of dual antiplatelet therapy after DES implantation in patients with or without acute coronary syndromes: an individual patient data pairwise and network meta-analysis of six randomized trials and 11473 patients. Eur. Heart. J. 21: 627 (2017).

3. Giustino G, Chieffo A, Palmerini T, et al. Efficacy and safety of Dual Antiplatelet Therapy After Complex PCI. J. Am. Coll. Cardiol. 68(17): 1851-1864 (2016).

4. Grines CL, BonowRO, Casey DE, et al. Prevention of premature discontinuation of dual antiplatelet therapy in patients with coronary artery stents: a science advisory from the American Heart Association, American College of Cardiology, Society for Cardiovascular Angiography and Interventions, American College of Surgeons, and American Dental Association, with representation from the American College of Physicians. J. Am. Dent. Assoc. 138: 652-655 (2007).
5. Lillis T, Ziakas A, Koskinas K, Tsirlis A, Giannoglou G. Safety of dental extractions during uninterrupted single or dual antiplatelet treatment. Am. J. Cardiol. 108(7): 964-967 (2011).

6. Cardiac Society of Australia and New Zealand. Guidelines for the management of antiplatelet therapy in patients with coronary stents undergoing non-cardiac surgery. Heart. Lung. Circ. 19(1): 2-10 (2010).

7. Darvish-Kazem S, Gandhi M, Marcucci M, Douketis JD. Perioperative management of antiplatelet therapy in patients with a coronary stent who need non-cardiac surgery: a systematic review of clinical practice guidelines. Chest. 8: 13 (2013).

8. Sciahbasi A, Patrizi R, Madonna M, et al. Successful thrombolysis in patients with subacute and late stent thrombosis. Can. J. Cardiol. 25(6): e213-214 (2009).

9. Steg PG, James SK, Atar D, et al. Task Force on the management of ST-segment elevation acute myocardial infarction of the European Society of Cardiology (ESC). ESC Guidelines for the management of acute myocardial infarction in patients presenting with STsegment elevation. Eur. Heart. J. 33(20): 2569-2619 (2012).

10. Papanikolaou J, Makris D, Tsolaki V, Spathoulas K, Zakynthinos E. Post-partum hemorrhage complicated by reverse-Takotsubo cardiogenic shock; a novel therapeutic approach. Am. J. Emerg. Med. 19: S735-S757 (2016). 